\title{
Effects on the emission discrepancy between two- dimensional Sn-based and Pb-based perovskites
}

\author{
Tianju Zhang (张天举) $)^{1,2}$, Chaocheng Zhou (周超成 $)^{3,4}$, Jia Lin (林 佳 $)^{3^{* *}}$, and Jun Wang $(\text { 王 俊 })^{1,2,5^{*}}$ \\ ${ }^{1}$ Laboratory of Micro-Nano Optoelectronic Materials and Devices, Shanghai Institute of Optics and Fine Mechanics, Chinese Academy of Sciences, \\ Shanghai 201800, China \\ ${ }^{2}$ Center of Materials Science and Optoelectronic Engineering, University of Chinese Academy of Sciences, Beijing 100049, China \\ 3 Department of Physics, Shanghai Key Laboratory of Materials Protection and Advanced Materials in Electric Power, Shanghai University of Electric Power, \\ Shanghai 200090, China \\ ${ }^{4}$ State Key Laboratory of Advanced Optical Communication Systems and Networks, School of Physics and Astronomy, Shanghai Jiao Tong University, \\ Shanghai 200240, China \\ ${ }^{5}$ CAS Center for Excellence in Ultra-Intense Laser Science, Shanghai 201800, China
}

*Corresponding author: jwang@siom.ac.cn

${ }^{* *}$ Corresponding author: jlin@shiep.edu.cn

Received October 12, 2021 | Accepted November 10, 2021 | Posted Online December 2, 2021

Two-dimensional (2D) Sn-based perovskites are a kind of non-toxic environment-friendly emission material with low photoluminescence quantum yields (PLQYs) and enhanced emission linewidths compared to that of 2D Pb-based perovskites. However, there is no work systematically elucidating the reasons for the differences in the emission properties. We fabricate (BA) ${ }_{2} \mathrm{Snl}_{4}$ and $(\mathrm{BA})_{2} \mathrm{Pbl}_{4}$ having different defect densities and different exciton-phonon scattering intensities. We also reveal that 2D Sn-based perovskites have stronger exciton-phonon scattering intensity and higher defects density, significantly broadening the emission linewidth and accelerating the exciton relaxation process, which significantly reduces the PLQY of 2D Sn-based perovskites.

Keywords: temperature-dependent photoluminescence spectra; transient absorption spectra; defect state; excitonphonon scattering; two-dimensional perovskites.

DOI: 10.3788/COL202220.021602

\section{Introduction}

Self-assembled two-dimensional (2D) Ruddlesden-Popper phase organic-inorganic halide perovskites with quantum-well structures have attracted much research attention in optoelectronic devices, lasers, and light-emitting diodes (LEDs) by virtue of their superior features such as enhanced stability, high absorption coefficient, tunable light-emitting wavelength, high color purity, and high exciton binding energy ${ }^{[1-10]}$. Its chemical structure formula is $\left(\mathrm{RNH}_{3}\right)_{2}(\mathrm{~A})_{n-1} \mathrm{~B}_{n} \mathrm{C}_{3 n+1}$, where $\mathrm{R}$ is an alkylor aromatic group, $\mathrm{A}$ is an organic cation, $\mathrm{B}$ is $\mathrm{Pb}^{2+}$, $\mathrm{Sn}^{2+}, \mathrm{Ge}^{2+}$, or other elements, and $\mathrm{C}$ is a halogen element ${ }^{[11]}$. For the $2 \mathrm{D}$ perovskites system with $n=1$, the organic cation layer's dielectric constant is smaller than that of the inorganic octahedral layer in combination with the inorganic layer's thickness of about $0.7 \mathrm{~nm}^{[12-14]}$, which causes the weakened dielectric screening effect and the enhanced spatial confinement effect. These effects greatly weaken the dielectric screening between electron-hole pairs to enhance the Coulomb interaction force between them within the inorganic layer, and thus the exciton dominates the optical transitions in materials ${ }^{[12,15-17]}$. Although 2D Pb-based perovskites are favored in the field of LEDs for display applications and lasers, the element $\mathrm{Pb}^{2+}$ is extremely toxic to the environment, limiting its further development. Compared to $\mathrm{Pb}^{2+}, \mathrm{Sn}^{2+}$ is non-toxic and has the most similar ionic radius ${ }^{[18]}$, so the lattice parameters obtained by $\mathrm{Sn}^{2+}$ replacement are, in principle, not severely modified ${ }^{[19,20]}$, which makes $\mathrm{Sn}$-based perovskites expected to replace $\mathrm{Pb}$-based perovskites in the future. At the same time, compared with the 2D Pb-based perovskites LEDs for display applications, the 2D Sn-based perovskites LEDs suffer from the disadvantages of low photoluminescence quantum yields (PLQYs) and enhanced emission linewidths [full width at half-maximum $(\mathrm{FWHM})>40 \mathrm{~nm}]^{[21,22]}$. Although much research points out that Sn-based perovskites are more likely to have a higher density of defect states, due to the oxidation potential of $\mathrm{Sn}^{2+} / \mathrm{Sn}^{4+}(-0.15 \mathrm{eV})$ being much lower than that of $\mathrm{Pb}^{2+} / \mathrm{Pb}^{4+}(-1.8 \mathrm{eV})^{[23]}$, and numerous optimization strategies 
proposed to improve their PLQY ${ }^{[20-22,24-26]}$, it is still difficult to improve their emission linewidths comparable to that of $\mathrm{Pb}$ based perovskites $(\mathrm{FWHM}<20 \mathrm{~nm})^{[3,5,20,27,28]}$. There are a variety of factors affecting the emission linewidth of the material, mainly the inhomogeneous broadening caused by the disordered nature of the crystal structure and exciton-defect scattering effect, as well as the homogeneous broadening caused by the electron-phonon scattering effect ${ }^{[29-31]}$. However, no research work exists so far to systematically study these effects affecting emission properties between $2 \mathrm{D} \mathrm{Pb}$-based perovskites and 2D Sn-based perovskites under the same experimental conditions and further reveal the differences between them.

In this work, we have fabricated $(\mathrm{A})_{2} \mathrm{SnI}_{4}$ and $(\mathrm{A})_{2} \mathrm{PbI}_{4}[\mathrm{~A}$ : nbutylammonium $\left.\left(\mathrm{CH}_{3}\left(\mathrm{CH}_{2}\right)_{3} \mathrm{NH}_{3}^{+}\left(\mathrm{BA}^{+}\right)\right)\right]$using the same method, which has different defect densities and different exciton-phonon scattering intensities. Taking advantage of the temperature-dependent photoluminescence (PL) spectra, transient absorption (TA) spectra, time-dependent PL relaxation kinetics, and PLQY experiments, we also revealed that 2D Sn-based perovskites have stronger exciton-phonon scattering intensity and higher defect-state density relative to $2 \mathrm{D}$ Pb-based perovskites, which lead to a significant broadening of the emission linewidth and accelerate the exciton relaxation process of 2D Sn-based perovskites, and these effects reduce the PLQY of 2D Sn-based perovskites. These results can guide further improvement in the emission performance of 2D Sn-based perovskites, in which we should select new structures for organic cation layers with relatively high rigidity to reduce the exciton-phonon scattering intensity and use antioxidants to reduce the defect-state density and thus the energy non-radiative loss.

\section{Results and Discussion}

To explain the physical mechanism affecting the optical properties of 2D Sn-based perovskites, we prepared $(\mathrm{BA})_{2} \mathrm{SnI}_{4}$ and (BA) ${ }_{2} \mathrm{PbI}_{4}$ films by the spin-coating method. The schematic of the hybrid quantum-well structure of $(\mathrm{BA})_{2} \mathrm{SnI}_{4}$ and $(\mathrm{BA})_{2} \mathrm{PbI}_{4}$ crystal structures [Fig. 1(a)] shows the perovskite octahedra sandwiched between organic spacer molecules $\left(\mathrm{BA}^{+}\right)$. Figure 1 shows the ultraviolet-visible (UV-Vis) absorption, steady-state PL spectroscopy, and PLQY of (BA) ${ }_{2} \mathrm{SnI}_{4}$ and $(\mathrm{BA})_{2} \mathrm{PbI}_{4}$. The inset shows that the optical bandgap of $(\mathrm{BA})_{2} \mathrm{SnI}_{4}$ and $(\mathrm{BA})_{2} \mathrm{PbI}_{4}$ was obtained as $1.97 \mathrm{eV}$ and $2.37 \mathrm{eV}$ by the Tauc-plot method, respectively. The PLQY of $(\mathrm{BA})_{2} \mathrm{SnI}_{4}$ is lower than that of $(\mathrm{BA})_{2} \mathrm{PbI}_{4}$, indicating that the defect density of $(\mathrm{BA})_{2} \mathrm{SnI}_{4}$ is higher than that of $(\mathrm{BA})_{2} \mathrm{PbI}_{4}$. Such defect states might arise from undesirable and uncontrolled conversion of $\mathrm{Sn}^{2+}$ to $\mathrm{Sn}^{4+}$, as the oxidation potential of $\mathrm{Sn}^{2+} / \mathrm{Sn}^{4+}(-0.15 \mathrm{eV})$, during the process of sample preparation in a glove box with almost the nitrogen environment, in which there is still a trace amount of oxygen that allows the $\mathrm{Sn}^{2+}$ to oxidize to $\mathrm{Sn}^{4+[22,23]}$. Besides, the oxidation potential of $\mathrm{Pb}^{2+} / \mathrm{Pb}^{4+}$ is $1.8 \mathrm{eV}$, which is more negative than that of $\mathrm{Sn}^{2+} / \mathrm{Sn}^{4+}$, making $\mathrm{Pb}^{2+}$ a stable state in $(\mathrm{BA})_{2} \mathrm{PbI}_{4}$. In addition, the FWHM of the normalized PL peak of $(\mathrm{BA})_{2} \mathrm{PbI}_{4}$ is a

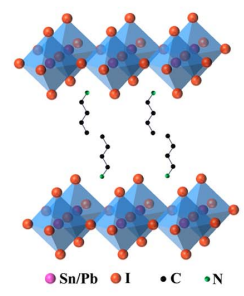

C

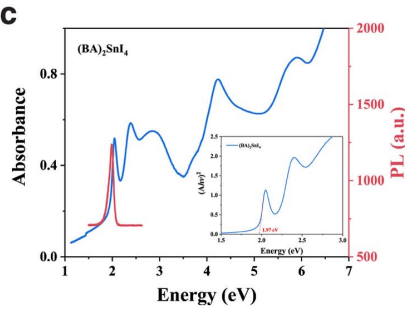

b

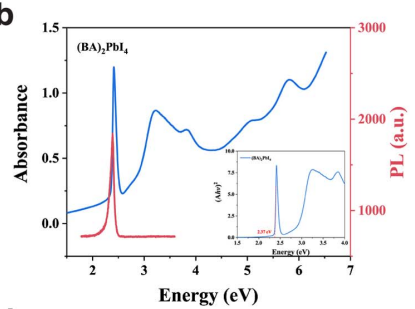

d

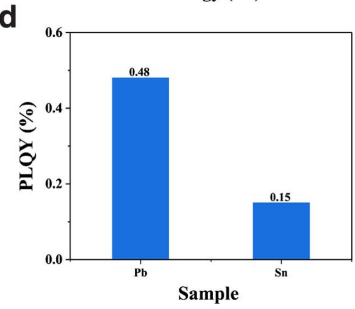

Fig. 1. Spectral characteristics of $(B)_{2} B_{4} \quad(B: S n / P b)$ perovskites. (a) Schematic of the hybrid quantum-well structure of the crystal structure, showing the perovskite octahedra sandwiched between organic spacer molecules $\left(\mathrm{BA}^{+}\right.$). The UV-visible (UV-Vis) absorption, steady-state photoluminescence (PL) spectra of (b) (BA) $)_{2} \mathrm{Snl}_{4}$ and (c) $(\mathrm{BA})_{2} \mathrm{Pbl} \mathrm{I}_{4}$. (d) PLQY of $(\mathrm{BA})_{2} \mathrm{Sn}_{4}$ and $(\mathrm{BA})_{2} \mathrm{Pbl}_{4}$

$18.5 \mathrm{~nm}$ smaller than that of $(\mathrm{BA})_{2} \mathrm{SnI}_{4}(39.2 \mathrm{~nm})$, as shown in Fig. 2. For 2D hybrid organic-inorganic perovskites, there is a trapping-state PL phenomenon due to its lattice being relatively soft. For the existence of two types of trap states, one is the intrinsic self-trapped exciton (STE) state caused by the exciton-phonon coupling, and the other trap state is the extrinsic STE state; the STE state is influenced by the local heterogeneity of the permanent defects lattice, which will sink to a different trapping depth ${ }^{[32-34]}$. These trap states can make the PL peaks deviate from the Gaussian line shape with PL tails in the broad spectral range at the low energy edge. To better fit the PL spectral
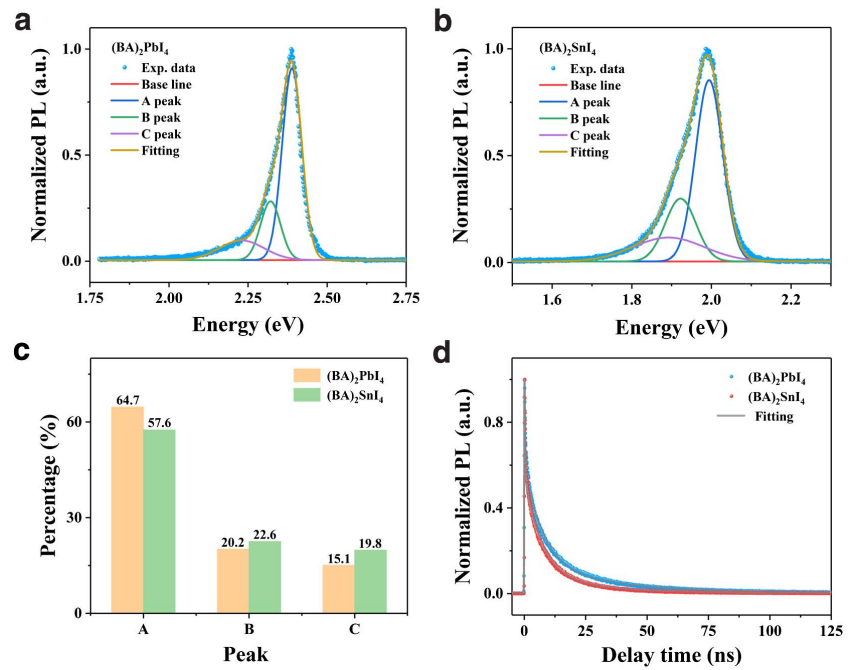

Fig. 2. Analysis of PL characteristics. (a) Multi-peak fit analysis of $\left.(B A)_{2}\right)_{2 b l}$ (b) multi-peak fit analysis of $(B)_{2} \mathrm{SnI}_{4}$ ( $(\mathrm{c})$ percentage of subpeaks of $(\mathrm{BA})_{2} \mathrm{Pbl}_{4}$ and $(B A)_{2} \mathrm{Snl}_{4,}(\mathrm{~d})$ normalized PL decay kinetics. 
line shapes of both materials, we used three subpeak fits, where the A peak is the PL of the free exciton, and peaks B and C are the PL of the trap states [Figs. 2(a) and 2(b)]. We found that the percentage of trapping-state $\mathrm{PL}$ for $(\mathrm{BA})_{2} \mathrm{SnI}_{4}$ is larger than that of trapping-state $\mathrm{PL}$ for $(\mathrm{BA})_{2} \mathrm{PbI}_{4}$, as shown in Fig. 2(c). At the same time, we have tested the time-resolved PL (TRPL) decay kinetics of the PL peak and further performed fitting using two decay exponents [Fig. 2(d)]. We found that the PL lifetime of (BA) ${ }_{2} \mathrm{PbI}_{4}$ is longer than that of $(\mathrm{BA})_{2} \mathrm{SnI}_{4}$, thus further evidencing that the PLQY of $(\mathrm{BA})_{2} \mathrm{PbI}_{4}$ is higher than that of (BA) ${ }_{2} \mathrm{SnI}_{4}$. In summary, we proved that (BA) ${ }_{2} \mathrm{SnI}_{4}$ has a higher defect density relative to (BA) ${ }_{2} \mathrm{PbI}_{4}$. For the $(\mathrm{BA})_{2} \mathrm{PbI}_{4}$, the valence band maximum (VBM) consists of an antibonding hybridization between $\mathrm{I}^{-}$(5p states) and $\mathrm{Pb}^{2+}$ (6s states), and the conduction band minimum (CBM) comprises a bonding hybridization between $\mathrm{Pb}^{2+}(6 \mathrm{p} \text { states })^{[23,35]}$. For the (BA) ${ }_{2} \mathrm{SnI}_{4}$, the VBM consists of an antibonding hybridization between $\mathrm{I}^{-}$ (5p states) and $\mathrm{Sn}^{2+}$ (5s states), and the CBM comprises a bonding hybridization between $\mathrm{Sn}^{2+}$ (5p states) ${ }^{[23]}$. The spin-orbit coupling of the $\mathrm{Sn}^{2+} 5 \mathrm{p}$ state is lower than that of the $\mathrm{Pb}^{2+}$ $6 \mathrm{p}$ state ${ }^{[36]}$, so this lowering of spin-orbit coupling increases the probability of defect states in (BA) ${ }_{2} \mathrm{SnI}_{4}$. In order to further analyze the factors affecting the FWHM, we conducted the temperature-dependent PL spectra to analyze the different physical processes of scattering (Fig. 3).

The variation of PL FWHM with temperature involves different physical processes of scattering, and the analysis of temperature-dependent PL FWHM is the main means to evaluate the electron-phonon coupling mechanism in various semiconductors ${ }^{[29,30]}$. Using first-order perturbation theory, the temperature-dependent FWHM can be approximately simplified to a model that contains four scattering mechanisms, as
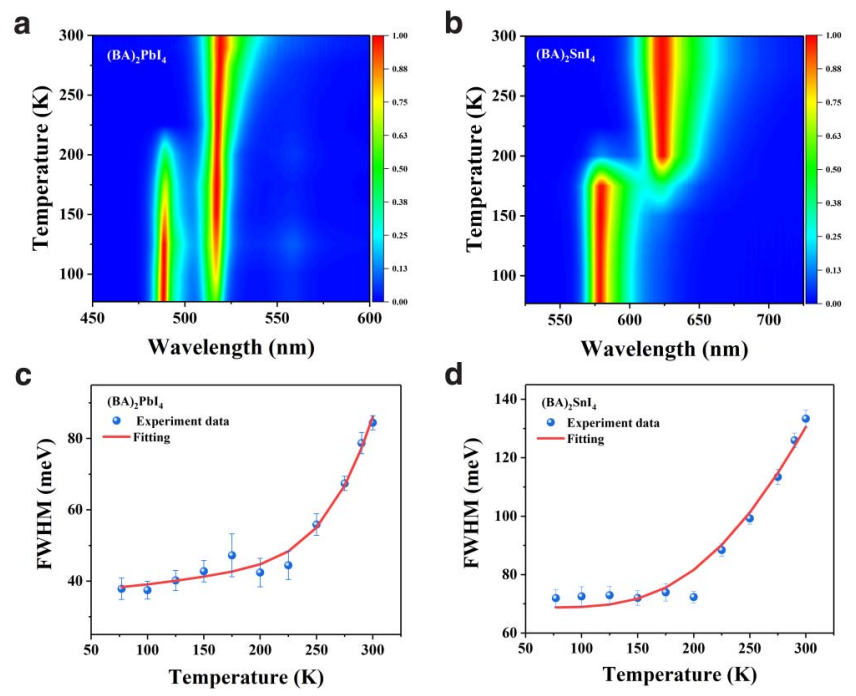

Fig. 3. Temperature dependence of the steady-state PL spectra. Contour map of the temperature-dependent steady-state $\mathrm{PL}$ spectra of (a) $(B A)_{2} \mathrm{Sn}_{4}$ and (b) $(\mathrm{BA})_{2} \mathrm{Pbl}_{4}$. Fitting of the temperature-dependent FWHM of (c) $(B A)_{2} \mathrm{Snl}_{4}$ and (d) $\left(\mathrm{BA}_{2} \mathrm{Pbl}_{4}\right.$. The red fitting line of the data (blue point) is obtained by Eq. (1). shown in Eq. (1) ${ }^{[37,38]}$. In the fitting process, the model utilizes a single phonon energy fit, which neglects the feature of the variation of phonon energy with temperature. Therefore, the fitting results have a certain limitation. However, it is helpful as a guide to understand the scattering between excitons and phonons in perovskite materials:

$$
\Gamma(T)=\Gamma_{0}+\gamma_{\mathrm{ac}} T+\frac{\Gamma_{\mathrm{LO}}}{e^{E_{\mathrm{LO}} / k_{B} T}-1}+\Gamma_{\mathrm{imp}} e^{-E_{\mathrm{imp}} / k_{B} T},
$$

where the first term $\Gamma_{0}$ on the right-hand side of Eq. (1) is the temperature-independent inhomogeneous broadening, which originates from the disordered nature of the crystal structure ${ }^{[29,30]}$. The second and third terms are temperaturedependent homogeneous broadening due to exciton-acoustic phonon scattering interactions and exciton-longitudinal optical (LO) phonon scattering interactions, where $\gamma_{\mathrm{ac}}$ is the coupling strength of the exciton-acoustic phonon scattering, $\Gamma_{\mathrm{LO}}$ is the Fröhlich coupling strength of the exciton scattering with LO phonons, and $E_{\mathrm{LO}}$ is the energy of the LO phonon. The last term describes the inhomogeneous broadening caused by ionized impurities (coupling $\Gamma_{\text {imp }}$ ), where the impurities with the binding energy, $E_{\text {imp }}$, are completely ionized. Since the FWHM gradient of increase is close to zero for both samples at low temperatures ${ }^{[38]}$, the exciton-acoustic phonon scattering effect can be excluded because its contribution can cause a linear growth characteristic of the FWHM at low temperatures, so $\gamma_{\mathrm{ac}}$ is equal to zero. This judgment is consistent with the results of polar inorganic semiconductors, i.e., acoustic phonon scattering is relatively negligible compared with the optical phonon scattering at room temperature ${ }^{[37-40]}$. The fitting parameters obtained by using the global fit optimization algorithm based on simulated annealing are shown in Table 1 . We can obtain from the fitting results that for $(\mathrm{BA})_{2} \mathrm{PbI}_{4}$, the optical phonon energy $E_{\mathrm{LO}}$ is $20.85 \pm 1 \mathrm{meV}$, and the exciton-optical phonon scattering intensity $\Gamma_{\mathrm{LO}}$ is $14.5 \pm 2 \mathrm{meV}$, which is in agreement with the results reported in the literature to indicate the rationality of the fitting results ${ }^{[38,39]}$. We found that the $\Gamma_{0}$ of $(\mathrm{BA})_{2} \mathrm{SnI}_{4}$ is almost twice as large as that of $(\mathrm{BA})_{2} \mathrm{PbI}_{4}$, demonstrating that the crystal structure of $(\mathrm{BA})_{2} \mathrm{SnI}_{4}$ is more disordered. Second, the exciton-optical phonon scattering intensity of $(\mathrm{BA})_{2} \mathrm{SnI}_{4}$ is much larger than that of $(\mathrm{BA})_{2} \mathrm{PbI}_{4}$, which greatly enlarges the PL FWHM of $(\mathrm{BA})_{2} \mathrm{SnI}_{4}$. Therefore, compared to $2 \mathrm{D} \mathrm{Pb}$-based perovskites, $2 \mathrm{D}$ Sn-based perovskites have higher crystal structure disorder and stronger exciton-optical phonon scattering intensities.

Table 1. Best-Fitting Parameters of the $(\mathrm{BA})_{2} \mathrm{Snl}_{4}$ and $(\mathrm{BA})_{2} \mathrm{Pbl}_{4}$ Perovskites.

\begin{tabular}{ccccc}
\hline Sample & $\Gamma_{0}(\mathrm{meV})$ & $\Gamma_{\mathrm{LO}}(\mathrm{meV})$ & $E_{\mathrm{LO}}(\mathrm{meV})$ & $E_{\text {imp }}(\mathrm{meV})$ \\
\hline$(\mathrm{BA})_{2} \mathrm{Sn}_{4}$ & $63.1 \pm 2$ & $192.8 \pm 4$ & $56.5 \pm 2$ & $94.9 \pm 2$ \\
$(\mathrm{BA})_{2} \mathrm{Pbl}_{4}$ & $37.7 \pm 1$ & $14.5 \pm 2$ & $20.85 \pm 1$ & $188.3 \pm 2$ \\
\hline
\end{tabular}


Femtosecond TA spectroscopy was utilized to further investigate the photophysical processes of the nonequilibrium interactions of photogenerated carriers ${ }^{[41-43]}$. The recombination characteristics of the photogenerated carriers in a material determine the emission properties of the material ${ }^{[44]}$. TA spectroscopy measurements of polycrystalline thin films prepared on quartz substrates were performed using our custom-built TA spectroscopy setup ${ }^{[45]}$. The light pulse generated by the Spectra-Physics Spirit laser (350 fs, $1 \mathrm{kHz}, 40 \mu \mathrm{J} / \mathrm{pulse}$ ) is split into two parts by an ultrafast beam splitter with a reflection to transmission energy ratio of 1:4. A frequency-doubled $520 \mathrm{~nm}$ output from the transmitted light was used as the pump beam, whereas the reflected laser pulse was used to excite a sapphire crystal for generating a white light continuous spectrum, whose chirp effect is corrected. The pump beam was chopped at $500 \mathrm{~Hz}$, and the probe beam transmitting the sample is collected by an ultrafast fiber optic spectrometer after passing through a short pass filter with a cutoff wavelength of $950 \mathrm{~nm}$. The time window of the TA measurement was $1.6 \mathrm{~ns}$. The spot radii of the pump light and probe light are determined to be $320 \pm 0.5$ and $10 \pm 0.5 \mu \mathrm{m}$, respectively, by the knife-cut method, and the pump light and probe light overlap at the same point of the sample in a noncollinear manner.

Figure 4 shows the false-color 2D TA mappings of the $(\mathrm{BA})_{2} \mathrm{SnI}_{4}$ and $(\mathrm{BA})_{2} \mathrm{PbI}_{4}$ thin films. For the TA spectra of (BA) ${ }_{2} \mathrm{SnI}_{4}$, there are photoinduced ground-state bleaching signals approximately at $610 \mathrm{~nm}$ and $518 \mathrm{~nm}$, which are caused by the band filling effect induced by pump light ${ }^{[46]}$. Within the first picosecond (ps), the interaction of hot excitons leads to the bandgap renormalization process, which in turn results in the signal of photoinduced absorption ${ }^{[47,48]}$. The photoinduced bleaching signals also exist in the broad spectral range $(650-750 \mathrm{~nm})$ below the bandgap, mainly caused by the filling of defect states with carriers excited by pump light. For the TA spectra of (BA) ${ }_{2} \mathrm{PbI}_{4}$, there is a photoinduced bleaching signal at about $518 \mathrm{~nm}$ caused by the band filling effect, while there is still a photoinduced bleaching signal in the wide range below the bandgap.

The relaxation process of photogenerated carriers at low excitation fluence of $3 \mu \mathrm{J} / \mathrm{cm}^{2}$ is analyzed by global fitting based on three relaxation decay components with different lifetimes ${ }^{[49]}$. The multi-exponential fitting of the photogenerated excitons is described in our previous work ${ }^{[45]}$. Among them, the first ultrafast relaxation component has a lifetime of about the order of a hundred femtoseconds. In the recent study of TA kinetics, the first fast component was attributed to the surface defect trapping process $(<1 \mathrm{ps})$ in $2 \mathrm{D}\left(\mathrm{CH}_{3}\left(\mathrm{CH}_{2}\right)_{8} \mathrm{NH}_{3}\right)_{2} \mathrm{PbBr}_{4}$ perovskite $^{[50]}$, and the surface trap state captured the exciton process (600 fs) for monolayer $\mathrm{MoS}_{2}$ materials ${ }^{[51]}$. So, the process is assigned to a defect trapping excitons process and a bandgap renormalization process. The second relaxation component with a lifetime of about the order of a hundred picoseconds is attributed to an interband exciton recombination process. The recombination rate of this process is affected by the excitation fluence, exciton-defect scattering effect, and exciton-phonon scattering effect ${ }^{[45,52,53]}$. The third process with the lifetime of a few nanoseconds is attributed to a defect-state-
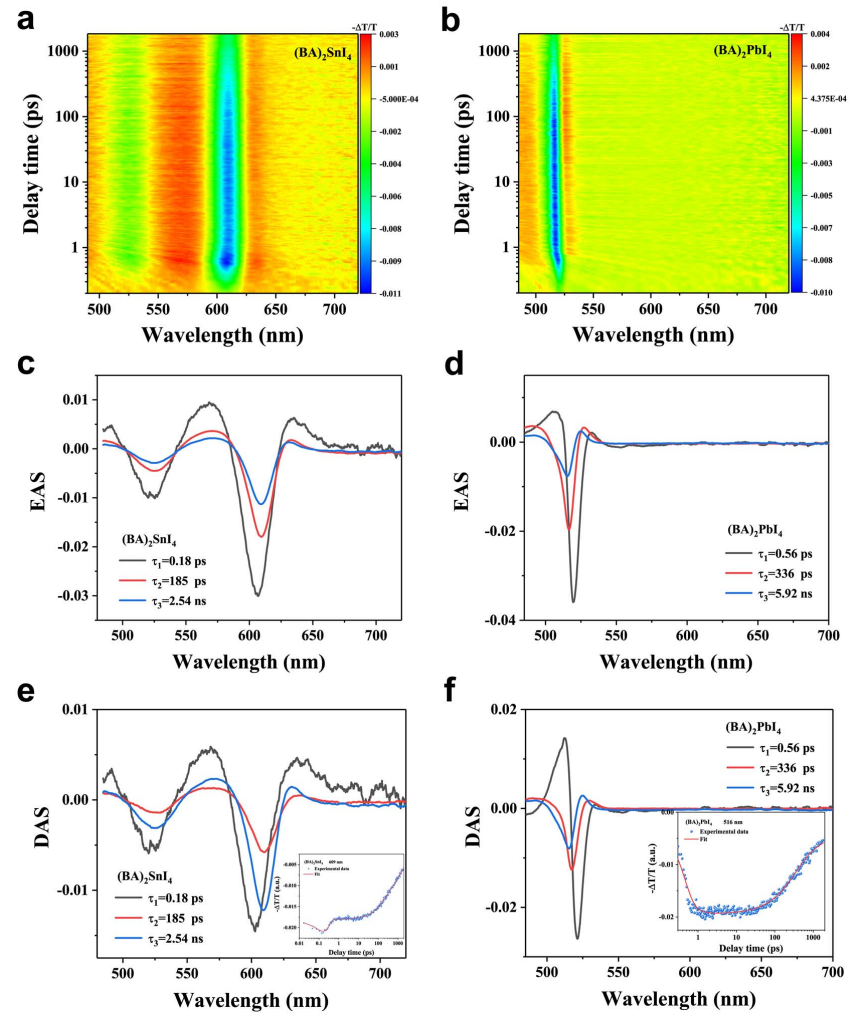

Fig. 4. Carrier relaxation dynamics. False-color 2D TA mappings of (a) $(B A)_{2} \mathrm{Snl}_{4}$ and (b) $\left(\mathrm{BA}_{2} \mathrm{Pbl}_{4}\right.$. Evolution-associated spectra (EAS) obtained upon global analysis of the TA data of (C) $(B A)_{2} \mathrm{Snl}_{4}$ and (d) $(B A)_{2} \mathrm{Pbl}_{4}$. Decay-associated spectra (DAS) obtained by performing global analysis on the TA spectra of (e) $(\mathrm{BA})_{2} \mathrm{Snl}_{4}$ and $(\mathrm{f})(\mathrm{BA})_{2} \mathrm{Pbl}_{4}$. The inset shows the results of fitting the band-edge exciton relaxation dynamics.

assisted recombination process ${ }^{[54,55]}$. The band-side exciton radiative relaxation recombination rate of $(\mathrm{BA})_{2} \mathrm{SnI}_{4}$ is faster compared with that of $(\mathrm{BA})_{2} \mathrm{PbI}_{4}$. This is mainly due to the stronger exciton-phonon scattering intensity and exciton-defect scattering intensity, which both accelerate the exciton recombination rate and reduce the percentage of exciton radiative recombination processes ${ }^{[33,56,57]}$. Tretiak et al. revealed that the more flexible geometry results in faster electron-hole recombination and shorter carrier lifetime, diminishing the PLQY for softer $2 \mathrm{D}$ perovskites by the density generalized function theory calculations ${ }^{[57]}$. Therefore, the soft organic cations [e.g., alkylammonium chain $\left.\left(\mathrm{BA}^{+}\right)\right]$can be replaced by relatively rigid structural organic cations [e.g., phenyl-ethylammonium $\left(\mathrm{C}_{6} \mathrm{H}_{5} \mathrm{CH}_{2} \mathrm{CH}_{2} \mathrm{NH}_{3}^{+}\left(\mathrm{PEA}^{+}\right)\right)$cation containing benzene rings] to limit their thermal movement between the inorganic octahedron layers and further reduce the exciton-phonon scattering intensity. At the same time, $\mathrm{PEA}^{+}$has a greater ability to hinder the formation of tin oxidation, where $\mathrm{BA}^{+}$ions increased the defect formation energy of $\mathrm{Sn}^{4+}$ by $0.33 \mathrm{eV}$, while $\mathrm{PEA}^{+}$could increase the defect formation energy of $\mathrm{Sn}^{4+}$ by $0.6 \mathrm{eV}^{[58]}$. Therefore, there is a necessity to find organic cation layers with the appropriate structure to further improve the emission characteristics of 2D Sn-based perovskites. 


\section{Conclusion}

In summary, we have fabricated samples $(\mathrm{A})_{2} \mathrm{SnI}_{4}$ and $(\mathrm{A})_{2} \mathrm{PbI}_{4}$ [A: n-butylammonium $\left(\mathrm{CH}_{3}\left(\mathrm{CH}_{2}\right)_{3} \mathrm{NH}_{3}^{+}\left(\mathrm{BA}^{+}\right)\right)$] using the same method and have revealed that $2 \mathrm{D}$ Sn-based perovskites have stronger exciton-phonon scattering intensity and higher defect density of states relative to $2 \mathrm{D} \mathrm{Pb}$-based perovskites by the temperature-dependent PL spectra, TA spectra, time-dependent PL relaxation kinetics, and PLQY experiments. These factors lead to a significant broadening of the emission linewidth and accelerate the exciton relaxation process, which reduces the PLQY of 2D Sn-based perovskites. Our results can be a guide for further improving the emission performance of 2D Sn-based perovskites by selecting new structures of organic cation layers with relatively high rigidity to reduce the exciton-phonon scattering intensity and by using antioxidants to reduce the defectstate density in the material and thus the energy loss of non-radiative transitions.

\section{Experimental Section}

\subsection{Syntheses of the $(B A)_{2} \mathrm{Snl}_{4}$ and $(\mathrm{BA})_{2} \mathrm{Pbl}_{4}$ perovskites polycrystalline thin films}

The glass substrate was cleaned sequentially with detergent, deionized water, ethanol, and isopropanol. Then, the substrate was treated with oxygen plasma for $10 \mathrm{~min}$ and dried in an argon flow. For the synthesis of $(\mathrm{BA})_{2} \mathrm{SnI}_{4}$ perovskite film, $0.1 \mathrm{mmol}$ $\mathrm{SnI}_{2}$ and $0.2 \mathrm{mmol}$ BAI were dissolved in $1 \mathrm{~mL}$ dimethyl formamidine (DMF) : dimethyl sulfoxide (DMSO) $(v: v=4: 1)$ to form the perovskite precursor solution, which was heated and stirred at $70^{\circ} \mathrm{C}$ for a few hours before use. Subsequently, the above-mentioned precursor solution was deposited on top of the glass substrate via a spin-coating process at $2500 \mathrm{r} / \mathrm{min}$ for $60 \mathrm{~s}$ in the argon-filled atmosphere. Then, the perovskite film was obtained after thermal annealing at $70^{\circ} \mathrm{C}$ for $5 \mathrm{~min}$. The fabrication procedure of $(\mathrm{BA})_{2} \mathrm{PbI}_{4}$ perovskite thin films is identical to that of $(\mathrm{BA})_{2} \mathrm{SnI}_{4}$.

\subsection{Temperature-dependent PL measurement}

For temperature-dependent PL measurement, polycrystalline thin films prepared on silica substrates were mounted in a cryostat (Janis ST-100) and cooled by liquid nitrogen. The samples were excited by the continuous wave (CW) laser excitation at a wavelength of $473 \mathrm{~nm}$, power density of $2 \mu \mathrm{J} / \mathrm{cm}^{2}$, and $25 \mathrm{~K}$ intervals. Fluorescence is separated by the $150 \mathrm{~g} / \mathrm{mm}$ grating in the Monochromator SP2500 of Princeton Instruments. Then, the spectral information was collected by the PIXIS100BX CCD at $-75^{\circ} \mathrm{C}$.

\subsection{Photoluminescence quantum yield}

PLQY of polycrystalline thin films prepared on silica substrates was measured using the Edinburgh FLS1000 instrument with an excitation wavelength of $520 \mathrm{~nm}$.

\subsection{UV/visible absorption}

UV-Vis absorption spectra of polycrystalline thin films prepared on glass substrates were collected by a Lambda 950 UV-Vis spectrometer.

\subsection{Time-resolved PL}

The TRPL kinetics was detected by a HORIBA DeltaFlex ultrafast time-resolved fluorescence spectrometer, where the excitation wavelength is $405 \mathrm{~nm}$ at $1 \mu \mathrm{J} / \mathrm{cm}^{2}$.

\section{Acknowledgement}

The work was supported by the National Natural Science Foundation of China (NSFC) (Nos. 61975221, 12174246, and 61875119), Strategic Priority Research Program of CAS (No. XDB16030700), and Shanghai Science and Technology International Cooperation Fund (No. 19520710200). The authors thank Dr. Chen from Instrumentation and Service Center for Molecular Sciences at Westlake University for supporting in the temperature-dependent steady-state PL spectral measurement and PLQY measurement.

\section{References}

1. F. Yuan, X. Zheng, A. Johnston, Y.-K. Wang, C. Zhou, Y. Dong, B. Chen, H. Chen, J. Z. Fan, G. Sharma, P. Li, Y. Gao, O. Voznyy, H.-T. Kung, Z.-H. Lu, O. M. Bakr, and E. H. Sargent, "Color-pure red light-emitting diodes based on two-dimensional lead-free perovskites," Sci. Adv. 6, eabb0253 (2020).

2. Y. Liu, J. Cui, K. Du, H. Tian, Z. He, Q. Zhou, Z. Yang, Y. Deng, D. Chen, X. Zuo, Y. Ren, L. Wang, H. Zhu, B. Zhao, D. Di, J. Wang, R. H. Friend, and Y. Jin, "Efficient blue light-emitting diodes based on quantum-confined bromide perovskite nanostructures," Nat. Photonics 13, 760 (2019).

3. X. Gong, O. Voznyy, A. Jain, W. Liu, R. Sabatini, Z. Piontkowski, G. Walters, G. Bappi, S. Nokhrin, O. Bushuyev, M. Yuan, R. Comin, D. McCamant, S. O. Kelley, and E. H. Sargent, "Electron-phonon interaction in efficient perovskite blue emitters," Nat. Mater. 17, 550 (2018).

4. A. G. Ricciardulli, S. Yang, J. H. Smet, and M. Saliba, "Emerging perovskite monolayers," Nat. Mater. 20, 1325 (2021).

5. M. Yuan, L. N. Quan, R. Comin, G. Walters, R. Sabatini, O. Voznyy, S. Hoogland, Y. Zhao, E. M. Beauregard, P. Kanjanaboos, Z. Lu, D. H. Kim, and E. H. Sargent, "Perovskite energy funnels for efficient light-emitting diodes," Nat. Nanotechnol. 11, 872 (2016).

6. Y. Wang, L. Song, Y. Chen, and W. Huang, "Emerging new-generation photodetectors based on low-dimensional halide perovskites," ACS Photonics 7, 10 (2019).

7. Z. L. Cao, F. R. Hu, C. F. Zhang, S. N. Zhu, M. Xiao, and X. Y. Wang, "Optical studies of semiconductor perovskite nanocrystals for classical optoelectronic applications and quantum information technologies: a review," Adv. Photon. 2, 054001 (2020).

8. J. Wu, R. Su, A. Fieramosca, S. Ghosh, J. Zhao, T. C. H. Liew, and Q. Xiong, "Perovskite polariton parametric oscillator," Adv. Photon. 3, 055003 (2021).

9. S. Hussain, A. Raza, F. Saeed, A. Perveen, Y. Sikhai, N. Din, E. E. Elemike, Q. Huang, A. Subramanian, Q. Khan, and W. Lei, "Stable and high performance all-inorganic perovskite light-emitting diodes with anti-solvent treatment," Chin. Opt. Lett. 19, 030005 (2021).

10. L. Jiang, X. Luo, Z. Luo, D. Zhou, B. Liu, J. Huang, J. Zhang, X. Zhang, P. Xu, and $\mathrm{G}$. $\mathrm{Li}$, "Interface and bulk controlled perovskite nanocrystal growth for 
high brightness light-emitting diodes [Invited]," Chin. Opt. Lett. 19, 030001 (2021).

11. K. Leng, I. Abdelwahab, I. Verzhbitskiy, M. Telychko, L. Chu, W. Fu, X. Chi, N. Guo, Z. Chen, Z. Chen, C. Zhang, Q. H. Xu, J. Lu, M. Chhowalla, G. Eda, and K. P. Loh, "Molecularly thin two-dimensional hybrid perovskites with tunable optoelectronic properties due to reversible surface relaxation," Nat. Mater. 17, 908 (2018).

12. D. B. Straus and C. R. Kagan, "Electrons, excitons, and phonons in twodimensional hybrid perovskites: connecting structural, optical, and electronic properties," J. Phys. Chem. Lett. 9, 1434 (2018).

13. J. C. Blancon, J. Even, C. C. Stoumpos, M. G. Kanatzidis, and A. D. Mohite, "Semiconductor physics of organic-inorganic 2D halide perovskites," Nat. Nanotechnol. 15, 969 (2020).

14. J. C. Blancon, A. V. Stier, H. Tsai, W. Nie, C. C. Stoumpos, B. Traore, L. Pedesseau, M. Kepenekian, F. Katsutani, G. T. Noe, J. Kono, S. Tretiak, S. A. Crooker, C. Katan, M. G. Kanatzidis, J. J. Crochet, J. Even, and A. D. Mohite, "Scaling law for excitons in 2D perovskite quantum wells," Nat. Commun. 9, 2254 (2018).

15. C. M. Mauck and W. A. Tisdale, "Excitons in 2D organic-inorganic halide perovskites," Trends Chem. 1, 380 (2019).

16. T. Palmieri, E. Baldini, A. Steinhoff, A. Akrap, M. Kollar, E. Horvath, L. Forro, F. Jahnke, and M. Chergui, "Mahan excitons in room-temperature methylammonium lead bromide perovskites," Nat. Commun. 11, 850 (2020).

17. M. Baranowski and P. Plochocka, "Excitons in metal-halide perovskites," Adv. Energy Mater. 10, 1903659 (2020).

18. D. H. Cao, C. C. Stoumpos, T. Yokoyama, J. L. Logsdon, T.-B. Song, O. K. Farha, M. R. Wasielewski, J. T. Hupp, and M. G. Kanatzidis, "Thin films and solar cells based on semiconducting two-dimensional RuddlesdenPopper $\left(\mathrm{CH}_{3}\left(\mathrm{CH}_{2}\right)_{3} \mathrm{NH}_{3}\right)_{2}\left(\mathrm{CH}_{3} \mathrm{NH}_{3}\right)_{n-1} \mathrm{Sn}_{n} \mathrm{I}_{3 n+1}$ perovskites," ACS Energy Lett. 2, 982 (2017)

19. F. Hao, C. C. Stoumpos, D. H. Cao, R. P. H. Chang, and M. G. Kanatzidis, "Lead-free solid-state organic-inorganic halide perovskite solar cells," Nat. Photonics 8, 489 (2014).

20. C. Gao, Y. Jiang, C. Sun, J. Han, T. He, Y. Huang, K. Yao, M. Han, X. Wang, Y. Wang, Y. Gao, Y. Liu, M. Yuan, and H. Liang, "Multifunctional naphthol sulfonic salt incorporated in lead-free $2 \mathrm{D}$ tin halide perovskite for red lightemitting diodes," ACS Photonics 7, 1915 (2020).

21. J. T. Lin, C. C. Liao, C. S. Hsu, D. G. Chen, H. M. Chen, M. K. Tsai, P. T. Chou, and C. W. Chiu, "Harnessing dielectric confinement on tin perovskites to achieve emission quantum yield up to $21 \%$," J. Am. Chem. Soc. 141, 10324 (2019).

22. Y. Liao, H. Liu, W. Zhou, D. Yang, Y. Shang, Z. Shi, B. Li, X. Jiang, L. Zhang, L. N. Quan, R. Quintero-Bermudez, B. R. Sutherland, Q. Mi, E. H. Sargent, and Z. Ning, "Highly oriented low-dimensional tin halide perovskites with enhanced stability and photovoltaic performance," J. Am. Chem. Soc. 139, 6693 (2017).

23. A. Swarnkar, V. K. Ravi, and A. Nag, "Beyond colloidal cesium lead halide perovskite nanocrystals: analogous metal halides and doping," ACS Energy Lett. 2, 1089 (2017).

24. Y. J. Heo, H. J. Jang, J.-H. Lee, S. B. Jo, S. Kim, D. H. Ho, S. J. Kwon, K. Kim, I. Jeon, J.-M. Myoung, J. Y. Lee, J.-W. Lee, and J. H. Cho, "Enhancing performance and stability of tin halide perovskite light emitting diodes via coordination engineering of Lewis acid-base adducts," Adv. Funct. Mater. 2106974 (2021).

25. Z. Wang, F. Wang, B. Zhao, S. Qu, T. Hayat, A. Alsaedi, L. Sui, K. Yuan, J. Zhang, Z. Wei, and Z. Tan, "Efficient two-dimensional tin halide perovskite light-emitting diodes via a spacer cation substitution strategy," J. Phys. Chem. Lett. 11, 1120 (2020)

26. Z. He, Y. Liu, Z. Yang, J. Li, J. Cui, D. Chen, Z. Fang, H. He, Z. Ye, H. Zhu, $\mathrm{N}$. Wang, J. Wang, and Y. Jin, "High-efficiency red light-emitting diodes based on multiple quantum wells of phenylbutylammonium-cesium lead iodide perovskites," ACS Photonics 6, 587 (2019).

27. M. D. Smith, B. A. Connor, and H. I. Karunadasa, "Tuning the luminescence of layered halide perovskites," Chem. Rev. 119, 3104 (2019).

28. M. C. Weidman, M. Seitz, S. D. Stranks, and W. A. Tisdale, "Highly tunable colloidal perovskite nanoplatelets through variable cation, metal, and halide composition," ACS Nano 10, 7830 (2016).

29. J. Lee, E. S. Koteles, and M. O. Vassell, "Luminescence linewidths of excitons in GaAs quantum wells below 150 K," Phys. Rev. B 33, 5512 (1986).
30. S. Rudin, T. L. Reinecke, and B. Segall, "Temperature-dependent exciton linewidths in semiconductors," Phys. Rev. B 42, 11218 (1990).

31. X. B. Zhang, T. Taliercio, S. Kolliakos, and P. Lefebvre, "Influence of electronphonon interaction on the optical properties of III nitride semiconductors," J. Phys. Condens. Mat. 13, 7053 (2001).

32. M. D. Smith and H. I. Karunadasa, "White-light emission from layered halide perovskites," Acc. Chem. Res. 51, 619 (2018).

33. S. Kahmann, E. K. Tekelenburg, H. Duim, M. E. Kamminga, and M. A. Loi, "Extrinsic nature of the broad photoluminescence in lead iodide-based Ruddlesden-Popper perovskites," Nat. Commun. 11, 2344 (2020).

34. E. P. Booker, T. H. Thomas, C. Quarti, M. R. Stanton, C. D. Dashwood, A. J. Gillett, J. M. Richter, A. J. Pearson, N. Davis, H. Sirringhaus, M. B. Price, N. C. Greenham, D. Beljonne, S. E. Dutton, and F. Deschler, "Formation of long-lived color centers for broadband visible light emission in low-dimensional layered perovskites," J. Am. Chem. Soc. 139, 18632 (2017).

35. B. Traore, L. Pedesseau, L. Assam, X. Che, J. C. Blancon, H. Tsai, W. Nie, C. C. Stoumpos, M. G. Kanatzidis, S. Tretiak, A. D. Mohite, J. Even, M. Kepenekian, and C. Katan, "Composite nature of layered hybrid perovskites: assessment on quantum and dielectric confinements and band alignment," ACS Nano 12, 3321 (2018).

36. A. Hermann, J. Furthmüller, H. W. Gäggeler, and P. Schwerdtfeger, "Spinorbit effects in structural and electronic properties for the solid state of the group-14 elements from carbon to superheavy element 114," Phys. Rev. B 82, 155116 (2010).

37. R. Saran, A. Heuer-Jungemann, A. G. Kanaras, and R. J. Curry, "Giant band gap renormalization and exciton phonon scattering in perovskite nanocrystals," Adv. Opt. Mater. 5, 1700231 (2016).

38. A. D. Wright, C. Verdi, R. L. Milot, G. E. Eperon, M. A. Perez-Osorio, H. J. Snaith, F. Giustino, M. B. Johnston, and L. M. Herz, "Electron-phonon coupling in hybrid lead halide perovskites," Nat. Commun. 7, 11755 (2016).

39. L. Ni, U. Huynh, A. Cheminal, T. H. Thomas, R. Shivanna, T. F. Hinrichsen, S. Ahmad, A. Sadhanala, and A. Rao, "Real-time observation of exciton-phonon coupling dynamics in self-assembled hybrid perovskite quantum wells," ACS Nano 11, 10834 (2017).

40. J. Li, J. Hu, J. Ma, X. Wen, and D. Li, "Identifying self-trapped excitons in 2D perovskites by Raman spectroscopy [Invited]," Chin. Opt. Lett. 19, 103001 (2021).

41. N. Mondal, A. De, S. Das, S. Paul, and A. Samanta, "Ultrafast carrier dynamics of metal halide perovskite nanocrystals and perovskite-composites," Nanoscale 11, 9796 (2019).

42. Z. Guo, Y. Wan, M. J. Yang, J. Snaider, K. Zhu, and L. B. Huang, "Long-range hot-carrier transport in hybrid perovskites visualized by ultrafast microscopy," Science 356, 59 (2017).

43. J. Chen, C. Zhang, X. Liu, L. Peng, J. Lin, and X. Chen, "Review on the study of carrier dynamic process in all-inorganic halide perovskites by photoluminescence measurement," Photon. Res. 9, 151 (2020).

44. L. M. Herz, "Charge-carrier dynamics in organic-inorganic metal halide perovskites," Annu. Rev. Phys. Chem. 67, 65 (2016).

45. T. Zhang and J. Wang, "Defect-enhanced exciton-exciton annihilation in monolayer transition metal dichalcogenides at high exciton densities," ACS Photonics 8, 2770 (2021)

46. J. S. Manser and P. V. Kamat, "Band filling with free charge carriers in organometal halide perovskites," Nat. Photonics 8, 737 (2014).

47. X. Wu, M. T. Trinh, and X. Y. Zhu, "Excitonic many-body interactions in two-dimensional lead iodide perovskite quantum wells," J. Phys. Chem. C 119, 14714 (2015).

48. X. Wu, M. T. Trinh, D. Niesner, H. Zhu, Z. Norman, J. S. Owen, O. Yaffe, B. J. Kudisch, and X. Y. Zhu, "Trap states in lead iodide perovskites," J. Am. Chem. Soc. 137, 2089 (2015).

49. Z. Guo, Y. Wan, M. Yang, J. Snaider, K. Zhu, and L. Huang, "Long-range hotcarrier transport in hybrid perovskites visualized by ultrafast microscopy," Science 356, 59 (2017).

50. X. L. Li, X. Lian, J. H. Pang, B. B. Luo, Y. H. Xiao, M. D. Li, X. C. Huang, and J. Z. Zhang, "Defect-related broadband emission in two-dimensional lead bromide perovskite microsheets," J. Phys. Chem. Lett. 11, 8157 (2020). 
51. H. Shi, R. Yan, S. Bertolazzi, J. Brivio, B. Gao, A. Kis, D. Jena, H. G. Xing, and L. Huang, "Exciton dynamics in suspended monolayer and few-layer $\mathrm{MoS}_{2}$ 2D crystals," ACS Nano 7, 1072 (2013).

52. Y. Jiang, M. Cui, S. Li, C. Sun, Y. Huang, J. Wei, L. Zhang, M. Lv, C. Qin, Y. Liu, and M. Yuan, "Reducing the impact of Auger recombination in quasi-2D perovskite light-emitting diodes," Nat. Commun. 12, 336 (2021).

53. Z. Guo, X. Wu, T. Zhu, X. Zhu, and L. Huang, "Electron-phonon scattering in atomically thin 2D perovskites," ACS Nano 10, 9992 (2016).

54. J. S. Yao, J. C. Zhang, L. Wang, K. H. Wang, X. C. Ru, J. N. Yang, J. J. Wang, X. Chen, Y. H. Song, Y. C. Yin, Y. F. Lan, Q. Zhang, and H. B. Yao, "Suppressing Auger recombination in cesium lead bromide perovskite nanocrystal film for bright light-emitting diodes," J. Phys. Chem. Lett. 11, 9371 (2020).
55. M. J. Trimpl, A. D. Wright, K. Schutt, L. R. V. Buizza, Z. Wang, M. B. Johnston, H. J. Snaith, P. Müller-Buschbaum, and L. M. Herz, "Charge-carrier trapping and radiative recombination in metal halide perovskite semiconductors," Adv. Funct. Mater. 30, 2004312 (2020).

56. K. Miyata, T. L. Atallah, and X. Y. Zhu, "Lead halide perovskites: crystalliquid duality, phonon glass electron crystals, and large polaron formation," Sci. Adv. 3, el701469 (2017).

57. D. Ghosh, A. J. Neukirch, and S. Tretiak, "Optoelectronic properties of twodimensional bromide perovskites: influences of spacer cations," J. Phys. Chem. Lett. 11, 2955 (2020).

58. A. Mahata, D. Meggiolaro, L. Gregori, and F. De Angelis, "Suppression of tin oxidation by 3D/2D perovskite interfacing," J. Phys. Chem. C 125, 10901 (2021). 